\title{
The Effect of Thiamine Treatment on the Activity of Pyruvate Dehydrogenase: Relation to the Treatment of Leigh's Encephalomyelopathy
}

\author{
F. A. Hommes ${ }^{[19]}$, R. Berger, and G. Luit-de-HaAn \\ Laboratory of Developmental Biochemistry Department of Pediatrics, University of Groningen \\ School of Medicine, Groningen, The Netherlands
}

\begin{abstract}
Extract
Rats received intraperitoneal injection of thiamine $(125 \mathrm{mg} / \mathrm{kg}$ body wt) for 4 consecutive days. There was less inhibition by ATP $(9 \% \pm 2 \%)$ of the pyruvate dehydrogenase complex of isolated liver mitochondria from treated rats than in liver mitochondria from control rats $(30 \% \pm 8 \%)$. The liver mitochondria of the treated animals contained about $25 \%$ more thiamine pyrophosphate than the mitochondria of the controls $(0.48 \pm 0.03$ and $0.36 \pm 0.01$ nmoles $/ \mathrm{mg}$ mitochondrial protein, respectively).

Roche and Reed have demonstrated inhibition of the phosphorylation of the pyruvate dehydrogenase complex by thiamine pyrophosphate, thereby maintaining pyruvate dehydrogenase in the active form. It is suggested that the therapeutic effect of high doses of thiamine given to patients who suffer from Leigh's disease is, at least in part, due to maintainance of the pyruvate dehydrogenase complex in its active form, thus facilitating the oxidation of pyruvate.
\end{abstract}

\section{Speculation}

Administration of high doses of thiamine to patients suffering from subacute necrotizing encephalomyelopathy seems to have a beneficial effect. Adverse effects, because of interference with a physiologic control mechanism, i.e., inhibition of the phosphorylation of the pyruvate dehydrogenase complex, may, however, interfere with its therapeutic value.

\section{Introduction}

Subacute necrotizing encephalomyelopathy or Leigh's disease [10] is an inborn error of metabolism [9] characterized by symmetrical lesions of the brainstem. Two theories on the cause of the disease have been proposed: the first based on the demonstration of a deficiency of pyruvate carboxylase [9] subsequently confirmed by Yoshida et al. [18], Delvin et al. [6], and Tang et al. [17] and the second based on a deficiency of thiamine triphosphate, caused by inhibition of thiamine diphosphate:ATP-phosphotransferase by specific inhibitors circulating in the body fluids of affected patients [3].

Thiamine given in high doses to a patient suffering from Leigh's disease does, at least temporarily, improve the clinical condition, while the high blood lactic acid and pyruvic acid concentrations decrease after administration of thiamine [5]. Concomitantly, the blood 
$\beta$-hydroxybutyrate and acetoacetate concentrations increased. These data have been interpreted as an activation of the pyruvate dehydrogenase complex by thiamine $[5,8]$.

Roche and Reed [14] have demonstrated recently that thiamine pyrophosphate inhibits the phosphorylation of the pyruvate dehydrogenase, thereby maintaining the pyruvate dehydrogenase complex in its active form. The question arises, therefore, as to whether administration of high doses of thiamine can maintain the pyruvate dehydrogenase complex in the nonphosphorylated active form. Rats receired intraperitoneal injections of high doses of thiamine. The activity of pyruvate dehydrogenase of liver mitochondria was then determined under phosphorylating and nonphosphorylating conditions. It was found that thiamine treatment of the rats resulted in less inhibition by ATP of liver pyruvate dehydrogenase as compared with controls.

\section{Materials and Methods}

Young adult, female rats of the Wistar strain of about $200 \mathrm{~g}$, body wt, received daily intraperitoneal injections of $\mathrm{I} \mathrm{ml}$ solution containing $25 \mathrm{mg}$ thiamine dichloride in $0.9 \% \mathrm{NaCl}$ for 4 consecutive clays. The animals were killed on the 5th day. Liver mitochondria were prepared in 0.25 м sucrose as described by Schneider [15]. The rate of oxygen uptake was measured polarographically [2] in a medium which had the following composition: $\mathrm{K}_{2} \mathrm{HPO}_{4}, 10 \mathrm{~mm}$; $\mathrm{KCl}, 15 \mathrm{~mm} ; \mathrm{MgCl}_{2}, 5 \mathrm{~mm}$; EDTA, 2 mu; trishydroxymethylaminomethane hydrochloride, $50 \mathrm{~mm}$; fluorocitrate to inhibit further oxidation of citrate, $0.1 \mathrm{~mm}$; carboxyl cyanide $p$-trifluoromethoxyphenyl hydrazone as an uncoupler, $1 \mu \mathrm{M}$; oligomycin to inhibit the mitochondrial ATPase, 6 $\mu \mathrm{g} / \mathrm{ml}$; malate to generate an adequate supply of oxaloacetate, $5 \mathrm{~mm}$; pyruvate, $0.5 \mathrm{~mm}$; ADP or ATP, $5 \mathrm{~mm}$.

Protein was determined by the biuret method, using bovine serum albumin as a standard.

Mitochondrial extracts were prepared with $18 \%$ $\mathrm{HClO}_{4}$, followed by neutralization with $30 \% \mathrm{KHCO}_{3}$. After standing for $1 \mathrm{hr}$ at $0^{\circ}$ the mixture was centrifuged and the supernatant analyzed for thiamine phosphates as described by Cooper et al. [3].

All reagents used were of analytical grade.

\section{Results}

Table I summarizes the results on the rates of oxygen uptake of rat liver mitochondria with pyruvate and
Table I. Rates of oxygen uptake by rat liver mitochondria with pyruvate and malate as substrate ${ }^{1}$

\begin{tabular}{|c|c|c|}
\hline $\begin{array}{l}\text { Treatment of animal: and } \\
\text { addition to basic medium }\end{array}$ & $\begin{array}{c}\text { Rate of } \mathrm{O}_{2} \text { uptake } \\
\text { in } n \text { at } \mathrm{O} / \mathrm{min} \cdot \mathrm{mg} \\
\text { protein }\end{array}$ & Inhibition, $\%$ \\
\hline \multicolumn{3}{|l|}{ No treatment } \\
\hline$\triangle D P, 5 \mathrm{mM}$ & 42.7 & \multirow{2}{*}{22} \\
\hline $\mathrm{ATP}, 5 \mathrm{~mm}$ & 33.3 & \\
\hline \multicolumn{3}{|l|}{ Thiamine treated $(1)$} \\
\hline ADP, $5 \mathrm{~mm}$ & 52.4 & \multirow[b]{2}{*}{7} \\
\hline$\triangle T P, 5 \mathrm{~mm}$ & 49.0 & \\
\hline \multicolumn{3}{|l|}{ Thiamine treated (2) } \\
\hline$\triangle D P, 5 \mathrm{~mm}$ & 51.6 & \multirow{2}{*}{9} \\
\hline$\triangle T P, 5 \mathrm{~mm}$ & 49.0 & \\
\hline \multicolumn{3}{|l|}{ None (2) } \\
\hline $\mathrm{ADP}, 5 \mathrm{~mm}$ & 61.6 & \multirow{2}{*}{37} \\
\hline$\triangle \mathrm{TP}, 5 \mathrm{~mm}$ & 38.8 & \\
\hline \multicolumn{3}{|l|}{ Thiamine treated (3) } \\
\hline$\triangle \mathrm{DP}, 5 \mathrm{~mm}$ & 67.5 & \multirow{2}{*}{12} \\
\hline$\triangle T P, 5 \mathrm{mM}$ & 59.5 & \\
\hline
\end{tabular}

1 The incubation medium, as well as the scheme of injection for thiamine treatment of the rats, is described under Materials and Methods. Mean inhibition \pm SE for control animals, $30 \% \pm 8 \%$; for thiamine-treated animals, $9 \% \pm 2 \%$.

${ }^{2}$ Number of animals receiving treatment is indicated in parenthesis.

malate as substrate, in the medium described under materials and methods in the presence of ADP or ATP.

In the presence of ADP and an uncoupler, the pyruvate dehydrogenase is maximally activated because the intramitochondrial ATP level is essentially zero [7]. The same experiment repeated in the presence of ATP and oligomycin to prevent the hydrolysis of ATP demonstrates inhibition of the rate of oxygen uptake. That the rate of oxygen uptake is directly related to the activity of pyruvate dehydrogenase in this system has been demonstrated by showing that the ratio of acetylCoA over CoA is decreased in the presence of ATP and that, furthermore, the rate of oxidation of palmitoyl carnithine is higher than that of pyruvate and malate $[1,16]$. Citrate synthase is, in this system, not rate limiting. Liver mitochondria prepared from thiaminetreated rats show considerably less inhibition of the pyruvate dehydrogenase by ATP than the nontreated controls (Table I). Administration of thiamine can apparently maintain the pyruvate dehydrogenase in the active, nonphosphorylated form, which may be expected from the observations of Roche and Reed [14]. Therefore, the mitochondria prepared from thiamine- 
Table II. Thiamine pyrophosphate content of liver mitochondria of rats with and without thiamine treatment ${ }^{1}$

\begin{tabular}{lc}
\hline Treatment of animal & $\begin{array}{c}\text { Thiamine pyrophosphate } \\
\text { content of liver mitochon- } \\
\text { dria in protein, nmol/mg }\end{array}$ \\
\hline None (1) & 0.37 \\
Thiamine treated (1) & 0.50 \\
Thiamine treated (2) & 0.50 \\
None (2) & 0.35 \\
Thiamine treated (3) & 0.43 \\
\hline
\end{tabular}

${ }^{1}$ Number of animals receiving treatment is indicated in parentheses. The mean value $\pm \mathrm{SE}$ for the control animals is $0.36 \pm$ 0.01 and for the treated animals is $0.48 \pm 0.03$.

treated rats have to contain more thiamine diphosphate than those of the controls.

Electrophoretic analysis of mitochondrial extracts at pH 5.2 demonstrated that the only fraction which could be made visible with the fluorescing ferricyanide reagent, had the same electrophoretic mobility as thiamine diphosphate. This was observed for control animals as well as for thiamine-treated animals. Subsequent analyses of mitochondrial extracts for thiamine derivatives were therefore carried out on whole extracts, without electrophoretic separation. The results are given in Table II. Liver mitochondria prepared from thiamine-treated rats contain significantly more thiamine diphosphate than do liver mitochondria prepared from control animals.

\section{Discussion}

Pincus and associates [12, 13] have advocated the use of thiamine in cases of Leigh's disease as suggested by Lonsdale [11]. That administration of thiamine has a beneficial effect for those patients seems to be well clocumented $[5,8,11-13]$. The rationale for this treatment seems to be the role of thiamine triphosphate in neurophysiologic processes [12]. However, the effect of thiamine treatment on the blood lactate and pyruvate level in cases of Leigh's disease suggested in addition a different mode of action of this vitamin. The decrease in blood lactate and pyruvate and the concomitant increase in $\beta$-hydroxybutyrate and acetoacetate observed after institution of the thiamine therapy could be explained by postulating an activation of the pyruvate dehydrogenase complex $[5,8]$. Thiamine pyrophosphate was found by Roche and Reed [14] to inhibit the phosphorylation of pyruvate dehydrogenase. A high intramitochondrial concentration of thiamine pyrophosphate will therefore maintain the pyruvate dehydrogenase in its active form.
Phenomenologically, this could amount to the same as postulating an activation of the enzyme complex. If this mechanism is also operative for the effect of thiamine in cases of Leigh's disease it is necessary to demonstrate that thiamine treatment results in a lower sensitivity to ATP inhibition of the pyruvate dehydrogenase complex. The data presented in Table I demonstrate that this is indeed the case. Table II demonstrates, furthermore, that treatment of the rats with high doses of thiamine results in an increased content of thiamine pyrophosphate in the mitochondria. It is therefore highly likely that the mechanism suggested by Reed and Roche [14] is also operative in the case of the thiamine-treated rats and consequently also in cases of Leigh's disease treated with high doses of thiamine.

The present findings do not exclude a role of thiamine triphosphate in the ethiology of the neuropathologic findings as observed in Leigh's disease $[3,4]$. The maintainance of the pyruvate dehydrogenase complex in its active form, as demonstrated here, is, however, an effect of the thiamine administration. How far interference by the thiamine therapy with a physiologic control mechanism has any adverse effects remains to be established in future investigations.

\section{Summary}

Intraperitoneal injection in rats of high doses of thiamine resulted in an increased content of thiamine pyrophosphate of the liver mitochondria. Concomitantly a decreased sensitivity to inhibition by ATP of liver pyruvate dehydrogenase was found. This result has been related to the earlier observed decrease in blood lactate and pyruvate after institution of thiamine therapy in patients suffering from Leigh's disease.

\section{References and Notes}

1. Berger, R., And Hommes, F. A.: Control of pyruvate oxidation by rat liver mitochondria, no. 1105. Abstracts of the Communications of the 8 th Meeting of the European Biochemical Society, Amsterdam, 1972.

2. Chance, B., And Williams, G. R.: A simple and rapid assay of oxidative phosphorylation. Nature, 175: 1120 (1955).

3. Cooper, J. R., Irokawa, Y., and Pincus, J. H.: Thiamine triphosphate deficiency in subacute necrotizing encephalomyelopathy. Science, 164: 72 (1969).

4. Cooper, J. R., Prncus, J. H., Itokawa, Y., and Piros, K.: Experience with phosphoryl transferase inhibition in subacute necrotizing encephalomyelopathy. New Engl. J. Med., 283: $793(1970)$.

5. De Groot, C. J., Jonxis, J. H. P., And Hommes, F. A.: In: J. Stern and C. Toothill: Further Studies on Leigh's En- 
cephalomyelopathy in organic acidaemias. Society for the Study of Inborn Errors of Metabolism, Symposium No. 9. (Churchill and Livingstone, Ltd., London, 1972). p. 40.

6. Delvin, E., Neol, J. L., and Scriver, C. R.: Pyruvate carboxylase: Two forms in human liver. Pediat. Res., 6: 392 (1972).

7. Held 5, H. W., Klingenibrg, M., and Milovancev, M.: Differences between ATP/ADP ratios in the mitochondrial matrix and in the extramitochondrial space. Eur. J. Biochem., 30: 434 (1972).

8. Hommes, F. A.: In: F. A. Hommes and C. J. Van den Berg: Biochemistry-Inborn Errors of Metabolism. (Academic Press, New York, in press).

9. Hommes, F. A., Polman, H. A., and Reerink, J. D.: Leigh's encephalomyelopathy: an inborn error of gluconeogenesis. Arch. Dis. Childhood, 43: 423 (1968).

10. Leigh, D.: Subacute necrotizing encephalomyelopathy in an infant. J. Neurol. Neurosurg. Psychiat., 14: 256 (1951).

11. LoNSdAle, D.: Hyperalaninaemia with pyruvicemia. New Engl. J. Med., 278: 1235 (1968).

12. Pincus, J. H.: Subacute necrotizing encephalomyelopathy (Leigh's diseasc): A consideration of clinical features and ctiology. Develop. Med. Childhood Neurol., 14: 87 (1972).

13. Puncus, J. H., Coorer, J. R., Itokawa, Y., and Gumbinos, M.:
Subacute necrotizing encephalomyelopathy. Arch. Neurol., 2f: $511(1971)$.

14. Roche, T. E., ANd ReEd, L. J.: Function of the nonidentical subunits of mammalian pyruvate dehydrogenase. Biochem. Biophys. Res. Commun., 48: 840 (1972).

15. Schneider, W. C.: Intracellular distribution of enzymes. III. The oxidation of octanoic acid by rat liver fractions. J. Biol. Chem., 176: 259 (1948).

16. Schuster, S. M., AND Olson, M. S.: Regulation of pyruvate oxidation in isolated rabbit heart mitochondria. J. Biol. Chem., 247: 5088 (1972).

17. TANG, T. T., Goon, T. A., Dyken, P. R., Johnson, S. D., McCready, S. R., Sy, S. T., LARdy, H. A., and Rudolph, F. B.: Pathogenesis of Leigh's encephalomyeolopathy. J. Pediat., 81 : 189 (1972).

18. Yoshida, T., Thda, K., Konno, T., and Arakawa, T.: Hyperalaninaemia with pyruvicaemia due to pyruvate carboxylase deficiency of the liver. Tohoku J. Exp. Med., 99: 121 (1969).

19. Requests for reprints should be addressed to: F. A. Hommes, Ph.D., Laboratory of Developmental Biochemistry, Department of Pediatrics, University of Groningen, Bloemsingel 10, Groningen, The Netherlands.

20. Accepted for publication February 20, 1973. 